\title{
Reply to the comment
}

\section{Matthias Schmautz $\cdot$ Niklas Lampenius}

We concur with Krotter/Schueler that neither the utilized Net Value Created (NVC) measure nor its adaptation to the FTE is new, however we never claimed it to be. We adopted the NVC to a non-life insurance company and indicated the contribution of the work of Schueler and Krotter through the reference Schueler and Krotter (2008) and the phrase "a term coined by Schueler and Krotter" when introducing the NPVtime effect. Our final result, a particular decomposition of the NVC based on a "cost of capital"-deviation, has not been disclosed and discussed by Schueler or Krotter.

\section{Refrences}

Schueler, A., Krotter, S.: The link between residual income and value created for levered firms: A note. Management Accounting Research. 19, 270-277 (2008)

M. Schmautz ( $\square)$

Köln, Germany

e-mail: schmautz@gmx.net

N. Lampenius

Stuttgart, Germany 